\title{
The effect of aged garlic extract on blood pressure and other cardiovascular risk factors in uncontrolled hypertensives: the AGE at Heart trial
}

This article was published in the following Dove Press journal:

Integrated Blood Pressure Control

27 January 2016

Number of times this article has been viewed

\section{Karin Ried ${ }^{1-3}$ \\ Nikolaj Travica' \\ Avni Sali'}

'National Institute of Integrative Medicine, Melbourne, ${ }^{2}$ Faculty of Health Science and Medicine, Bond University, Gold Coast, ${ }^{3}$ Department of General Practice, University of Adelaide, Adelaide, Australia
Correspondence: Karin Ried National Institute of Integrative Medicine, 2 I Burwood Road, Hawthorn, Melbourne, VIC 3I22, Australia

Tel +61399129545

Fax +6I 398040513

Email karinried@niim.com.au
Background: Hypertension affects $30 \%$ of adults worldwide. Garlic supplements have shown promise in the treatment of uncontrolled hypertension, and the mechanism of action is biologically plausible. Our trial is the first to assess the effect of aged garlic extract on central blood pressure and arterial stiffness, regarded as important risk factors for cardiovascular morbidity.

Subjects and methods: A total of 88 general practice patients and community members with uncontrolled hypertension completed a double-blind randomized placebo-controlled trial of 12 weeks investigating the effect of daily intake of aged garlic extract ( $1.2 \mathrm{~g}$ containing $1.2 \mathrm{mg}$ $S$-allylcysteine) or placebo on blood pressure, and secondary outcome measures of centralhemodynamics and other cardiovascular markers, including cholesterol, homocysteine, platelet function, and inflammatory markers.

Results: Mean blood pressure was significantly reduced by $5.0 \pm 2.1 \mathrm{mmHg}(P=0.016)$ systolic, and in responders by $11.5 \pm 1.9 \mathrm{mmHg}$ systolic and $6.3 \pm 1.1 \mathrm{mmHg}$ diastolic compared to placebo $(P<0.001)$. Central hemodynamic-measures tended to improve in the garlic group more than in the placebo group, including central blood pressure, central pulse pressure, mean arterial pressure, augmentation pressure, pulse-wave velocity, and arterial stiffness. While changes in other cardiovascular markers did not reach significance due to small numbers in subgroups with elevated levels, trends in beneficial effects of garlic on the inflammatory markers TNF $\alpha$, total cholesterol, low-density lipid cholesterol, and apolipoproteins were observed. Aged garlic extract was highly tolerable and acceptable, and did not increase the risk of bleeding in patients on blood-thinning medication.

Conclusion: Our trial suggests that aged garlic extract is effective in reducing peripheral and central blood pressure in a large proportion of patients with uncontrolled hypertension, and has the potential to improve arterial stiffness, inflammation, and other cardiovascular markers in patients with elevated levels. Aged garlic extract was highly tolerable with a high safety profile as a stand-alone or adjunctive antihypertensive treatment.

Keywords: hypertension, central blood pressure, arterial stiffness, cardiovascular risk factors, aged garlic extract

\section{Introduction}

Hypertension affects 1 billion (one in four) adults worldwide, and attributes to about $40 \%$ of cardiovascular-related deaths. ${ }^{1-3}$ Standard antihypertensive medication is not always effective, leaving about $24 \%$ ( 3 million) of the adult population uncontrolled hypertensive. ${ }^{4}$ Garlic supplements have been associated with a blood pressure (BP)-lowering effect of clinical significance in hypertensive patients. ${ }^{5-8}$ The mechanism of action is biologically plausible, whereby garlic's BP-lowering effect 
involves the hydrogen sulfide- and nitric oxide-signaling pathways. ${ }^{9}$ Garlic in the form of Kyolic aged garlic extract is particularly effective and tolerable with a high safety profile, and standardized by dosage of the active ingredient $S$-allylcysteine (SAC). ${ }^{6,10}$

While previous research has shown aged garlic extract to reduce peripheral $\mathrm{BP}, 5-8$ this study is the first to assess the effect of aged garlic extract on central hemodynamic measures, including central BP, central pulse pressure, pulsewave velocity (PWV), and arterial stiffness.

Central hemodynamic measures and arterial stiffness are regarded as more important predictors or risk factors than peripheral BP for cardiovascular disease. ${ }^{11}$ Furthermore, arterial stiffness, an indicator of the loss of flexibility or hardening of the arteries, increases with age through loss of intact elastin and collagen fibers in the arterial wall, leading to atherosclerosis and contributing to increased BP. ${ }^{12}$ However, arterial stiffness is one of many other risk factors contributing to hypertension.

Here, we describe the effect and tolerability of aged garlic extract as an adjunct treatment on peripheral (office/clinical) BP, central hemodynamic measures, and cardiovascular markers in patients with uncontrolled hypertension.

\section{Subjects and methods Trial design and methods}

This study was approved by the Human Research Ethics Committee at National Institute of Integrative Medicine, and the trial was registered with the Australian New Zealand Clinical Trial Registry (ACTRN12613000747729). Participants provided informed written consent.

Adults with uncontrolled hypertension (systolic BP $[\mathrm{SBP}] \geq 140 \mathrm{mmHg}$ and/or diastolic $\mathrm{BP}[\mathrm{DBP}] \geq 90 \mathrm{mmHg}$ ) were sought to participate in the double-blind randomized placebo-controlled parallel 12-week trial investigating the effect of aged garlic extract on BP and other cardiovascular markers/parameters. We recruited through seven general practices in metropolitan Melbourne, Australia, as well as by distribution locally of flyers, postcards, advertising in the local newspaper, our institute's website, and social media. We included patients with a mean $\mathrm{SBP} \geq 138 \mathrm{mmHg}$ or mean DBP $\geq 85 \mathrm{mmHg}$ under clinical trial conditions who had been either on an established plan of prescription antihypertensive medication for at least 2 months or did not take any BP medication and their doctor did not plan to change their BP-medication regime during the trial. We excluded patients with unstable or serious conditions, including dementia, terminal illness, secondary hypertension, or pregnancy.
Patients were also excluded if they were not able to give informed consent or were taking daily supplements containing aged garlic extract.

\section{Allocation and treatment}

Consenting eligible patients were randomly allocated to the garlic or placebo group using a computer-generated permuted random-number table provided by an independent consulting statistician. Patients were assigned either two capsules daily of Kyolic aged garlic extract (Reserve formula; Wakunaga of America Co Ltd, Mission Viejo, CA, USA) ${ }^{13}$ containing $1.2 \mathrm{~g}$ of aged garlic extract powder and $1.2 \mathrm{mg} \mathrm{SAC}$ ) or to two placebo capsules daily for 12 weeks.

Kyolic aged garlic extract powder is manufactured from organically grown garlic bulbs that have undergone a 20-month natural aging process at room temperature. During the aging process, volatile sulfur components found in raw garlic, such as allicin, are chemically converted into stable and standardizable components, including the main active component SAC. ${ }^{13,14}$

Placebo capsules were matched in appearance to the active capsules, and packaged in identical containers by independent staff not involved in the trial. Activated carbon sachets were added to each container to disguise any odor.

Patients, investigators, and research assistants were blinded to treatment allocation. Blinding success of patients was assessed at the end of the trial by questionnaire. Patients were instructed to take the trial capsules in the evening with food. Patients were reminded to take their usual prescription medication as instructed by their doctor. Compliance was assessed by daily entries in calendars provided. Baseline demographics, exercise, and stress levels were assessed at the first appointment by questionnaire.

\section{Assessments}

\section{Blood pressure monitoring \\ Clinical blood pressure}

Primary outcome measures were SBP and DBP at 4,8 , and 12 weeks compared with baseline. BP was measured by a trained research assistant using two independent devices: 1) a calibrated and validated digital sphygmomanometer (HEM-907; Omron Corporation, Kyoto, Japan), and 2) an oscillometric ambulatory BP monitor (Mobil-O-Graph; IEM GmbH, Stolberg, Germany), with appropriately sized brachial cuffs.

The displays of the BP monitors were positioned away from the patient to assure blinding to the BP readings. BP measurements were taken with the patient in a seated position 
with the arm supported at heart level, after 5 minutes' rest, and after abstinence from food, caffeinated beverages, and smoking for a minimum of 2 hours prior to BP measurement at approximately the same time and day of the week. BP taken with the digital sphygmomanometer was recorded as three serial measurements at intervals of 30 seconds on both arms. Subsequently, BP was recorded with the Mobil-O-Graph device once on both arms, starting with the same arm as before. The mean of the BP measured with both devices on both arms was used in the analysis. If a BP reading deviated by more than $10 \mathrm{mmHg}$ from the average reading, the BP reading on that arm was repeated.

\section{Central blood pressure, arterial stiffness}

With the Mobil-O-Graph device, we also assessed central hemodynamic measures, including central BP, PWV, pulse pressure, and arterial stiffness at baseline and 4, 8, and 12 weeks. The Mobil-O-Graph uses brachial oscillometric BP waves for a noninvasive estimation of central BP, by taking a 10 -second snapshot of the radial arterial pressure wave and calculating the ascending aortic pressure wave with the ARCSolver algorithm, which in turn provides central BP, aortic augmentation index, ejection duration, and subendocardial viability ratio. ${ }^{15}$ The Mobil-O-Graph has been validated for automated BP monitoring against invasive recordings using benchmark solid-state pressure sensortipped catheters (Millar Instruments, Houston, TX, USA) and against a validated US Food and Drug Administrationapproved noninvasive system (SphygmoCor; AtCor Medical Inc., Sydney, Australia). ${ }^{16,17}$

Aortic PWV is considered the gold standard in the assessment of arterial stiffness, ${ }^{11}$ and can be measured noninvasively by brachial oscillometry or radial tonometry using the Mobil-O-Graph monitor. ${ }^{18}$ Measures of arterial stiffness, including augmentation pressure, augmentation index, and PWV, are strongly correlated with age and sex. ${ }^{19,20}$

\section{Cardiovascular biomarkers}

Fasted blood samples were taken by a research nurse at baseline and at 12 weeks to assess cardiovascular biomarkers, including serum cholesterol and triglycerides, lipoproteins, homocysteine, platelet function, and the inflammatory markers of ultrasensitive CRP, TNF $\alpha$, and IL-1 $\beta$. Platelet function was measured by clotting time on epinephrine/collagen and adenosine diphosphate/collagen using the PFA-100 platelet-function analyzer (Siemens AG, Munich, Germany), indicating platelet adhesion, activation, and aggregation. Principally, results indicate the longer the closure time the thinner the blood, and the shorter the closure time the thicker or stickier the blood. Liver and kidney function and glucose levels were also assessed by standard pathology assays.

\section{Tolerability and acceptability}

The tolerability of trial supplements was monitored throughout the trial by questionnaire at the 4-weekly appointments, and long-term acceptability was assessed at 12 weeks using our previously developed questionnaire. ${ }^{5,6}$ Patients were followed up by phone to assess reasons for withdrawal.

\section{Sample size}

A sample size of 100 patients was calculated based on the following assumptions: 1) to detect a difference of $10 \mathrm{mmHg}$ SBP (standard deviation 10) or $6.5 \mathrm{mmHg}$ DBP (standard deviation 10) in BP change between the active treatment $(n=50)$ and control groups $(n=50)$ with a power of $\geq 80 \%$ and $95 \%$ confidence; and 2 ) to account for $20 \%$ dropout or nonattendance at all appointments. Based on the experience of our previous trials, ${ }^{5,6}$ assuming a response rate of $15 \%$ and $50 \%$ of patients meeting eligibility criteria under trial conditions, we aimed to invite 1,630 patients from seven general practices.

\section{Statistical analysis}

Analyses were performed using IBM SPSS version 22. Statistical significance was set at $P<0.05$. Descriptive analysis was carried out for baseline characteristics. Differences between the groups were assessed by $\chi^{2}$ test or Fisher's exact test for binominal variables, Mann-Whitney $U$ test for ordinal variables, and one-way analysis of variance with Bonferroni adjustment for continuous variables. Potential confounding variables were included in the analysis using analysis of covariance (ANCOVA), eg, age and sex relevant for some central hemodynamic measures, and any baseline variables significantly different between groups. Primary outcome measures were clinical and central BP, and secondary outcome measures were other hemodynamic measures and cardiovascular markers.

Repeated-measure ANCOVA was used to assess the mean differences between groups for variables with multiple time points at 4,8 , and 12 weeks compared to baseline, including SBP and DBP and other central hemodynamic measures. Primary analysis was conducted with all participants following the protocol, excluding data points owing to BP-medication change and participant noncompliance of less than $50 \%$. 
Exploratory subgroup analysis using ANCOVA adjusted for baseline differences was performed by response to treatment for the primary outcome measure-BP. We defined responders to garlic treatment as mean reduction by more than $3 \%$ in SBP ( $\geq 5 \mathrm{mmHg}$ ) or DBP ( $\geq 3 \mathrm{mmHg}$ ) over time compared to baseline, which is clinically and statistically meaningful and similar to definitions by others. ${ }^{21}$

Exploratory subgroup analysis of secondary outcome measures using ANCOVA adjusted for age and sex was done by baseline levels (elevated versus normal) of selected central hemodynamic measures (eg, PWV, augmentation pressure, and augmentation index), and selected cardiovascular blood markers (total cholesterol, low-density lipoprotein [LDL], apolipoprotein A/B (ApoA/B), homocysteine, and plateletfunction markers).

\section{Results}

\section{Participants}

The trial was conducted in Melbourne, Australia between September 2013 and August 2014. Two-thirds of the patients with uncontrolled hypertension on medical record were recruited from seven metropolitan general practices with the support of eleven doctors. A third of the patients were recruited by letterbox drop of flyers, postcard displays at local pharmacies, advertising in the local newspaper, and through our institute's website and social media. Of the 1,170 invitations sent through general practices, $13 \%$ responded. A total of 185 patients were screened for eligibility, and 104 patients (56\%) were enrolled in the trial and randomly allocated to the garlic or the placebo group. Nine patients withdrew after their baseline assessment, due to personal reasons unrelated to the trial (Figure 1). After assessment of brachial BP and central hemodynamic measures using the digital sphygmomanometer and the ambulatory BP monitor (Mobil-O-Graph), eligible enrolled patients were asked to return to the clinic within the next few days for a fasted blood sample. On the day of the baseline blood-sample test, patients were provided with a month's supply of the trial medication and a calendar, until the next appointment in 4 weeks' time.

Baseline characteristics were not significantly different between groups, including BP medication and other prescription medication (eg, blood-thinning medication, lipidlowering medication, hormone-replacement therapy), as well as physical activities and stress levels (Table 1).

Of the 95 participants who completed the trial, seven were excluded due to significant protocol deviation known to influence primary outcome measures, including BP-medication

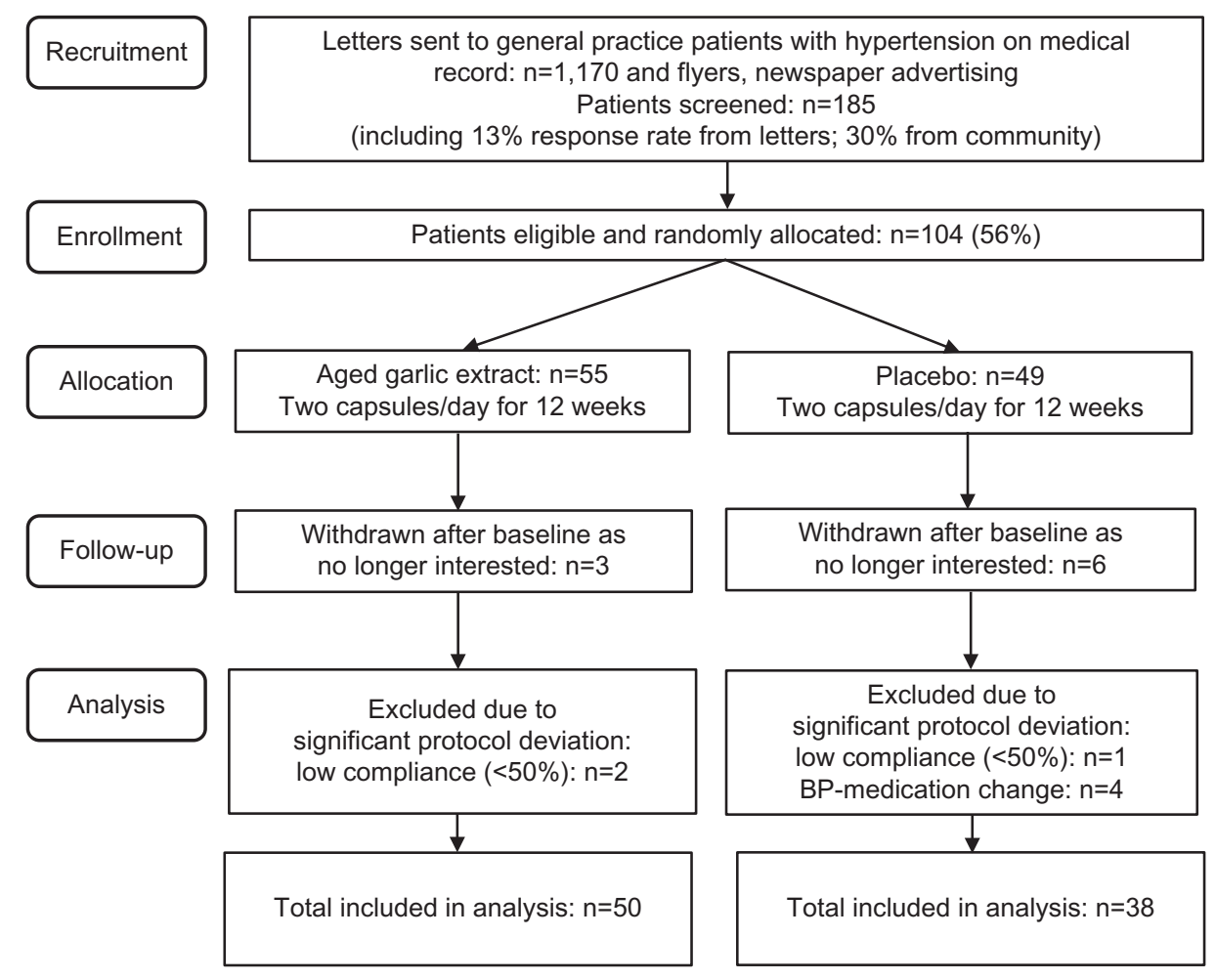

Figure I Trial flowchart.

Abbreviation: BP, blood pressure. 
Table I Baseline characteristics

\begin{tabular}{|c|c|c|c|c|c|}
\hline Demographics & All $(n=88)$ & Placebo $(n=38)$ & Garlic $(n=50)$ & $\begin{array}{l}\text { Garlic subgroup } \\
\text { responders }(n=29)\end{array}$ & $\begin{array}{l}\text { Garlic subgroup } \\
\text { nonresponders }(n=2 I)\end{array}$ \\
\hline & Mean \pm SD & & & & \\
\hline \multicolumn{6}{|l|}{ Age } \\
\hline Years & $62.3 \pm 11.3$ & $61.5 \pm 13.0$ & $63.3 \pm 9.9$ & $63.2 \pm 11.7$ & $63.4 \pm 8.2$ \\
\hline \multicolumn{6}{|l|}{ BMI } \\
\hline $\mathrm{kg} / \mathrm{m}^{2}$ & $27.7 \pm 4.9$ & $28.3 \pm 4.9$ & $27.3 \pm 4.9$ & $26.7 \pm 5.0$ & $28.1 \pm 4.8$ \\
\hline \multicolumn{6}{|l|}{ Vigorous exercise } \\
\hline Days/week & $1.4 \pm 2.0$ & $1.1 \pm 1.5$ & $1.6 \pm 2.3$ & $1.1 \pm 1.5$ & $1.3 \pm 2.1$ \\
\hline \multicolumn{6}{|l|}{ Moderate exercise } \\
\hline Days/week & $3.6 \pm 2.4$ & $3.5 \pm 2.4$ & $3.6 \pm 2.4$ & $3.5 \pm 2.4$ & $3.5 \pm 2.5$ \\
\hline \multicolumn{6}{|l|}{ Stress score (Cohen) } \\
\hline Range $0-40$ points & $10.1 \pm 6.6$ & $12.0 \pm 6.5$ & $13.9 \pm 6.6$ & $13.8 \pm 6.5$ & $13.9 \pm 6.7$ \\
\hline \multicolumn{6}{|l|}{ Other stressors } \\
\hline \multirow[t]{2}{*}{ Range $0-4$} & $0.4 \pm 0.8$ & $0.4 \pm 0.6$ & $0.5 \pm 0.9$ & $0.5 \pm 0.9$ & $0.5 \pm 0.9$ \\
\hline & n (\%) & & & & \\
\hline \multicolumn{6}{|l|}{ Sex } \\
\hline Male/female & $47 / 4 I(53 / 47)$ & $19 / 19(50 / 50)$ & $28 / 22(56 / 44)$ & $17 / \mid 2(59 / 4 \mid)$ & $10 / 11(48 / 52)$ \\
\hline Current smoker & $4(5)$ & $3(8)$ & I (2) & I (3) & 0 \\
\hline Family history of CVD & $56(64)$ & $26(68)$ & $30(60)$ & $17(59)$ & $13(62)$ \\
\hline Heart attack & $20(23)$ & $10(26)$ & $9(18)$ & $4(14)$ & $5(23)$ \\
\hline Stroke & $13(15)$ & $5(13)$ & $7(14)$ & $4(14)$ & $3(14)$ \\
\hline CAD, bypass & $13(15)$ & $3(8)$ & $10(20)$ & $5(17)$ & $5(23)$ \\
\hline Hypertension & $13(15)$ & $8(2 I)$ & $5(10)$ & $3(10)$ & $2(10)$ \\
\hline \multicolumn{6}{|l|}{ BP medication } \\
\hline Yes & $63(72)$ & $26(68)$ & $37(74)$ & $23(79)$ & $14(66)$ \\
\hline \multicolumn{6}{|l|}{ BP medications, $n$} \\
\hline 0 & $25(28)$ & $12(32)$ & $13(26)$ & $6(2 I)$ & $7(33)$ \\
\hline I & $35(40)$ & $12(32)$ & $23(46)$ & $15(52)$ & $8(38)$ \\
\hline 2 & $21(24)$ & II (29) & $10(20)$ & $5(17)$ & $5(24)$ \\
\hline 3 & $6(7)$ & $2(5)$ & $4(8)$ & $3(10)$ & $\mathrm{I}(5)$ \\
\hline 4 & $I(1)$ & I (3) & 0 & 0 & 0 \\
\hline \multicolumn{6}{|l|}{ BP-medication type } \\
\hline ACEI & $25(28)$ & II (29) & $14(28)$ & $10(34)$ & $4(19)$ \\
\hline A2RA & $34(39)$ & $15(40)$ & $19(38)$ & $12(4 I)$ & $7(33)$ \\
\hline $\mathrm{CCB}$ & $22(25)$ & $10(26)$ & $12(24)$ & $7(24)$ & $5(24)$ \\
\hline BB & $8(9)$ & $4(\mathrm{II})$ & $4(8)$ & $10(34)$ & $2(9)$ \\
\hline $\mathrm{D}$ & $14(16)$ & $7(18)$ & $7(14)$ & $3(10)$ & $4(19)$ \\
\hline \multicolumn{6}{|l|}{ Other medication } \\
\hline Yes & $4 \mid(47)$ & $23(60)$ & $18(36)$ & $9(3 I)$ & 9 (39) \\
\hline Blood-thinning medication & $22(25)$ & $10(20)$ & $12(32)$ & $6(21)$ & $4(19)$ \\
\hline Lipid (statin) & $15(17)$ & $7(18)$ & $8(16)$ & $2(7)$ & $6(29)$ \\
\hline Diabetes & $6(7)$ & $I(2)$ & $5(10)$ & $2(7)$ & $3(14)$ \\
\hline Depression/SSRI & $7(8)$ & $2(5)$ & $5(10)$ & $2(7)$ & $3(14)$ \\
\hline Reflux/PPI & $7(8)$ & $4(11)$ & $3(6)$ & $\mathrm{I}(\mathrm{II})$ & $2(10)$ \\
\hline Thyroid & $3(3)$ & $3(8)$ & 0 & 0 & 0 \\
\hline HRT (\% female) & $3(3)$ & $2(11)$ & I (2) & 0 & I (9) \\
\hline
\end{tabular}

Note: Garlic subgroup responders = reduction in systolic BP/diastolic BP $\geq 5 / 3 \mathrm{mmHg}$ over time.

Abbreviations: ACEl, angiotensin Il-converting enzyme inhibitor; A2RA, angiotensin Il-receptor antagonist; BB, $\beta$-blocker; BP, blood pressure; BMI, body mass index; CAD, coronary artery disease; CCB, calcium-channel blocker; CVD, cardiovascular disease; D, diuretic; HRT, hormone-replacement therapy; PPI, proton-pump inhibitor; SD, standard deviation; SSRI, selective serotonin-reuptake inhibitor.

change during the trial $(\mathrm{n}=4)$ and low compliance $(<50 \%$, $\mathrm{n}=3$ ), resulting in 88 participants for full analysis.

A total of 50 participants were allocated to the garlic group, and 38 participants to the placebo group, with a mean age of $62 \pm 12$ years and almost even distribution of sexes. The average body mass index in the trial participants was slightly overweight with a mean of $27.7 \pm 5 \mathrm{~kg} / \mathrm{m}^{2}$. Participants exercised moderately an average of 3.5 days a week (eg, 30 minutes of brisk walking, dancing, gardening, or housework) and 1.5 days vigorously (30 minutes of running, 
fast cycling, fast swimming, heavy shoveling, or carrying heavy loads). The average stress levels of participants assessed by the Cohen Stress score ${ }^{22}$ were comparable to the population average in a similar age-group.

Family history of cardiovascular events was reported by two-thirds of the participants, including myocardial infarction by almost $25 \%$ and stroke by $15 \%$ (Table 1 ). Three-quarters of participants were taking standard BP medication, with the majority taking one to two different types, with angiotensin II-receptor antagonists and angiotensin-converting enzyme inhibitors the most often prescribed, followed by calciumchannel blockers, diuretics, and $\beta$-blockers. Almost half of the participants took other prescription medication, including blood thinners (25\%) and lipid-lowering drugs (17\%), and a few took medication for diabetes, depression, reflux, thyroid issues, or hormone-replacement therapy (Table 1).

\section{Blood pressure and central hemodynamic measures}

Analysis of all participants $(n=88)$ revealed a significant reduction in SBP from baseline in the garlic group compared with placebo over 12 weeks (mean difference in SBP \pm standard error $=-5.0 \pm 2.1 \mathrm{mmHg}, P=0.016)$, but not for DBP (mean difference in $\mathrm{DBP} \pm$ standard error $=-1.9 \pm 1.2, P=0.12$; Table 2 and Figure 2).

About two-thirds of participants presented with high SBP or DBP at baseline, with about half of the participants having essential hypertension ( $\mathrm{SBP} \geq 140$ and DBP $\geq 90$ ). Subgroup analysis of participants with high SBP/DBP at baseline did not change results appreciably (Table 2).

However, closer evaluation of participants in the garlic group revealed that a proportion of participants $(50 \%-60 \%)$ responded to treatment over time. We defined responders to garlic treatment as mean reduction by more than 3\% in SBP ( $\geq 5 \mathrm{mmHg}$ ) or DBP ( $\geq 3 \mathrm{mmHg}$ ) over time compared to baseline, which is clinically and statistically meaningful and similar to definitions by others, ${ }^{21}$ while mean BP, assessed by repeated-measure ANCOVA, did not change appreciably for nonresponders. Subgroup analysis of responders revealed an average reduction of $11.5 \pm 1.9 \mathrm{mmHg} \mathrm{SBP}$ and $6.3 \pm 1.1 \mathrm{DBP}$ compared to placebo ( $P<0.001$; Table 2 and Figure 2$)$.

A number of central hemodynamic measures tended to improve more in the garlic group compared to placebo, including central SBP (mean difference $=-3.8 \pm 1.9 \mathrm{mmHg}, P=0.05$ ), central pulse pressure (mean difference $=-2.7 \pm 1.5 \mathrm{mmHg}$, $P=0.08$ ), total vascular resistance (mean difference $=-0.07 \pm 0.03$ $\mathrm{s} \cdot \mathrm{mmHg} / \mathrm{mL}, P=0.06$ ), mean arterial pressure (mean difference $=-3.1 \pm 1.8 \mathrm{mmHg}, P=0.09$ ), augmentation pressure if high at baseline (mean difference $=-3.5 \pm 1.8 \mathrm{mmHg}, P=0.06$ ), and $P W V$ if high at baseline (mean difference $=-0.33 \pm 0.14 \mathrm{~m} / \mathrm{s}$, $P=0.02$ ) (Table 2). Trends were confirmed in ANCOVA analysis including age and sex as covariates.

\section{Blood tests}

None of the blood markers tested changed significantly over time; however, lipid levels, including total cholesterol and LDL, tended to improve slightly more in the garlic group (Table 3). Subgroup analysis by lipid-lowering medication (eg, statins) intake did not change results appreciably. ApoA levels were highly correlated with high density lipoprotein (HDL) levels (Pearson $r=0.588, P<0.001$ ), and ApoB levels were highly correlated with LDL levels (Pearson $r=0.767$, $P<0.001)$, as expected. Reduction of ApoB when above the reference range was greater in the garlic group compared to placebo, albeit not statistically significant due to small sample size (mean difference $=-29.1 \pm 46.3 \mathrm{mg} / \mathrm{dL}, P=0.3$ $[\mathrm{n}=12])$. The average homocysteine level within participants in both groups was higher than the reference range (mean $14.2 \pm 3.6 \mu \mathrm{mol} / \mathrm{L})$, indicating potential underlying vitamin $\mathrm{B}_{12}$ or folate deficiencies.

Platelet-function analyses indicated a daily trial dosage of aged garlic extract to be safe, as platelet-aggregation times did not further increase in participants with slow platelet adhesion/aggression at baseline, often due to blood-thinning medication (eg, warfarin, aspirin). In contrast, platelet function tended to change more toward normal levels in the garlic group with high closure times (thin blood) at baseline for both agonists compared to the placebo group (Table 3 ).

The inflammatory marker TNF $\alpha$ was reduced in the garlic group compared to placebo with borderline significance $(P=0.05)$, while changes in IL-1 $\beta$ were not significant, but greater reduction was observed in the garlic group (Table 3). Subgroup analyses excluding patients with high inflammatory markers due to acute infection did not change results appreciably (data not shown). Neither glucose levels nor kidney-function and liver-function test variables changed noticeably over time within or between groups (Table 4).

\section{Tolerability, acceptability, and blinding}

Compliance of included participants was high in both groups (96.6\% $\pm 5.6 \%$ ). About $40 \%$ of participants in both groups reported minor side effects, including reflux (8\%), burping $(5 \%)$, bloating (3\%), and also improved digestion $(7 \%)(P>0.05$; Table 5). Few participants found side effects bothersome, and usually experienced these only in the first week of the trial. All but four participants found it easy (95\%) and acceptable (100\%) 
Table 2 Blood pressure and central hemodynamic measures

\begin{tabular}{|c|c|c|c|c|c|c|c|c|c|c|c|c|c|c|}
\hline \multicolumn{3}{|c|}{ Measures } & \multirow[t]{2}{*}{ Group } & \multirow[t]{2}{*}{$\mathbf{n}$} & \multicolumn{2}{|c|}{ Baseline } & \multirow{2}{*}{$\begin{array}{l}\begin{array}{l}\text { Baseline } \\
\text { difference }\end{array} \\
P \text {-value }\end{array}$} & \multicolumn{2}{|c|}{ I 2 weeks } & \multicolumn{2}{|c|}{ Change } & \multicolumn{3}{|c|}{$\begin{array}{l}\text { Mean difference between } \\
\text { groups (garlic vs placebo) }\end{array}$} \\
\hline \multirow[t]{25}{*}{$\begin{array}{l}\text { Office } \\
\text { BP }\end{array}$} & Variable & Details & & & Mean & SD & & Mean & SD & Mean & SD & $\begin{array}{l}\text { Mean } \\
\text { difference }\end{array}$ & SE & $P$-value \\
\hline & SBP & $\mathrm{mmHg}$ & Placebo & 38 & 142 & 9.4 & & 140.3 & 18.2 & -2.9 & 7.9 & & & \\
\hline & & & Garlic & 50 & 148.7 & 15.3 & 0.02 & $14 \mid .7$ & 15.3 & -8 & 10.6 & -5 & 2.1 & 0.02 \\
\hline & $\mathrm{SBP}>138 \mathrm{mmHg}$ & $\begin{array}{l}68 \% \text { high at } \\
\text { baseline }\end{array}$ & Placebo & 26 & 146.4 & 7.5 & & $|4| .4$ & 9.4 & -4.9 & 7 & & & \\
\hline & & $\begin{array}{l}76 \% \text { high at } \\
\text { baseline }\end{array}$ & Garlic & 38 & 153.8 & 13.8 & 0.02 & 143.8 & 12.1 & -10 & 10.8 & -5.1 & 2.4 & 0.04 \\
\hline & $\mathrm{SBP}<138 \mathrm{mmHg}$ & & Placebo & 12 & 132.4 & 4.5 & & 133.8 & 7.7 & 1.4 & 8.1 & & & \\
\hline & & & Garlic & 12 & 132.4 & 4.2 & NS & 130.9 & 7.1 & -1.5 & 6.6 & -2.9 & 3 & 0.34 \\
\hline & SBP placebo & & Placebo & 38 & 142 & 9.4 & & 139.1 & 9.5 & -2.9 & 7.9 & & & \\
\hline & SBP responders & $\begin{array}{l}58 \%>5 \mathrm{~mm} \\
\text { change }\end{array}$ & Garlic & 29 & 153.2 & 15.4 & 0.001 & 138.8 & 11.4 & -14.4 & 8.8 & -11.5 & 1.9 & $<0.001$ \\
\hline & SBP nonresponders & & Garlic & 21 & 142.3 & 12.9 & NS & 143.3 & 13.3 & I & 4.5 & 3.9 & 2.1 & 0.18 \\
\hline & DBP & & Placebo & 38 & 87.8 & 9.1 & & 86 & 9 & -1.8 & 5 & & & \\
\hline & & & Garlic & 50 & 89.9 & 11.7 & NS & 86.1 & 11.3 & -3.7 & 5.9 & -1.9 & 1.2 & 0.12 \\
\hline & $\mathrm{DBP}>85 \mathrm{mmHg}$ & $\begin{array}{l}63 \% \text { high at } \\
\text { baseline }\end{array}$ & Placebo & 24 & 92.9 & 6.6 & & 91.3 & 9.4 & -2.3 & 5.7 & & & \\
\hline & & $\begin{array}{l}64 \% \text { high at } \\
\text { baseline }\end{array}$ & Garlic & 32 & 96.2 & 9 & NS & 92.4 & 9.9 & -4.4 & 6.2 & -2.1 & & 0.19 \\
\hline & $\mathrm{DBP}<85 \mathrm{mmHg}$ & & Placebo & 14 & 79.1 & 5.1 & & 78.5 & 7.5 & -1 & 3.5 & & & \\
\hline & & & Garlic & 18 & 78.5 & 5.7 & NS & 76.7 & 7.7 & -2.3 & 5 & -1.3 & 1.6 & 0.40 \\
\hline & DBP placebo & & Placebo & 38 & 87.8 & 9.1 & & 86 & 9 & -1.8 & 5 & & & \\
\hline & DBP responders & $\begin{array}{l}50 \%>3 \mathrm{~mm} \\
\text { change }\end{array}$ & Garlic & 25 & 93.2 & $\mathrm{II} .4$ & NS & 85.1 & 10.9 & -1.8 & 3.8 & -6.3 & I.I & $<0.001$ \\
\hline & DBP nonresponders & & Garlic & 25 & 86.5 & II.I & NS & 88.4 & 12.7 & 0.8 & 4 & 2.5 & I.I & 0.08 \\
\hline & MAP & $\mathrm{mmHg}$ & Placebo & 38 & 112.7 & 10.6 & & 110.4 & 8.8 & -2.3 & 7.2 & & & \\
\hline & & & Garlic & 49 & 117.8 & 12.1 & 0.045 & 112.4 & 9.8 & -5.4 & 9.3 & -3.1 & 1.8 & 0.1 \\
\hline & $H R$ & $\mathrm{~L} / \mathrm{min}$ & Placebo & 38 & 70.4 & 9.6 & & 70 & 8.5 & -0.5 & 6 & & & \\
\hline & & & Garlic & 48 & 68.7 & 11.2 & NS & 67.9 & 10.7 & -0.7 & 6.6 & -0.2 & 1.4 & 0.9 \\
\hline & PP & $\mathrm{mmHg}$ & Placebo & 38 & 51 & 9.3 & & 49.8 & 9.2 & -1.3 & 9.5 & & & \\
\hline & & & Garlic & 49 & 54.2 & 14.7 & NS & 50.2 & 10.1 & -4 & 9.7 & -2.7 & 2.1 & 0.2 \\
\hline \multirow{13}{*}{$\begin{array}{l}\text { Central } \\
\text { BP }\end{array}$} & cSBP & $\mathrm{mmHg}$ & Placebo & 38 & 129.3 & 9.3 & & 126.8 & 9.9 & -2.4 & 7.8 & & & \\
\hline & & & & & & & & & & & & & & \\
\hline & & & Garlic & 48 & 134.8 & 14.2 & 0.04 & 128.5 & 10.3 & -6.2 & 9.7 & -3.8 & 1.9 & 0.05 \\
\hline & cDBP & $\mathrm{mmHg}$ & Placebo & 38 & 90.8 & 9.7 & & 88.6 & 8.8 & -2.2 & 6.3 & & & \\
\hline & & & Garlic & 48 & 95.1 & 12.3 & 0.08 & 90.4 & 10.7 & -4.7 & 8 & -2.6 & 1.6 & 0.11 \\
\hline & cPP & $\mathrm{mmHg}$ & Placebo & 38 & 38.4 & 7.3 & & 37.8 & 7.7 & -0.5 & 6.4 & & & \\
\hline & & & Garlic & 49 & 40.6 & 11.8 & NS & 37.5 & 8 & -3.2 & 7.4 & -2.7 & 1.5 & 0.08 \\
\hline & Cardiac output & $\mathrm{L} / \mathrm{min}$ & Placebo & 38 & 5.3 & 0.6 & & 5 & 0.5 & -0.3 & 0.5 & & & \\
\hline & & & Garlic & 49 & 5.1 & 0.7 & NS & 5.1 & 0.7 & -0.1 & 0.5 & 0.2 & 0.1 & 0.17 \\
\hline & TVR & $\mathrm{s} \cdot \mathrm{mmHg} / \mathrm{mL}$ & Placebo & 38 & 1.3 & 0.17 & & 1.33 & 0.15 & 0.04 & 0.13 & & & \\
\hline & & & Garlic & 49 & 1.41 & 0.21 & NS & 1.39 & 0.15 & -0.03 & 0.17 & -0.07 & 0.03 & 0.06 \\
\hline & Cardiac index & $\mathrm{L} / \mathrm{min} \times \mathrm{L} / \mathrm{m}^{2}$ & Placebo & 38 & 2.71 & 0.29 & & 2.58 & 0.33 & -0.13 & 0.25 & & & \\
\hline & & & Garlic & 49 & 2.65 & 0.45 & NS & 2.57 & 0.35 & -0.06 & 0.32 & 0.07 & 0.06 & 0.28 \\
\hline \multicolumn{15}{|c|}{ Arterial stiffness } \\
\hline & $\mathrm{AP}$ & $\mathrm{mmHg}$ & Placebo & 38 & 10.1 & 6.5 & & 10.8 & 6.6 & 0.76 & 5 & & & \\
\hline & & & Garlic & 49 & 13.2 & 8.9 & 0.07 & 11.6 & 4.9 & -1 & 5.6 & -1.9 & 1.2 & 0.12 \\
\hline & High AP* & $\mathrm{mmHg}$ & Placebo & 14 & 15.4 & 6.9 & & 14.4 & 8 & -1 & 5.3 & & & \\
\hline & & & Garlic & 23 & 20.6 & 7.5 & NS & 15.2 & 3 & -4.6 & 5.1 & -3.5 & 1.8 & 0.06 \\
\hline & High AP, male & $\mathrm{mmHg}$ & Placebo & 2 & 10 & 2.8 & & 7.5 & 0 & 0.3 & 3.3 & & & \\
\hline & & & Garlic & 7 & 23.1 & 10.5 & NS & 15 & 7.1 & -6.3 & 4.2 & -6.6 & 2.7 & 0.04 \\
\hline
\end{tabular}


Table 2 (Continued)

\begin{tabular}{|c|c|c|c|c|c|c|c|c|c|c|c|c|c|c|}
\hline \multicolumn{3}{|c|}{ Measures } & \multirow[t]{2}{*}{ Group } & \multirow[t]{2}{*}{$\mathbf{n}$} & \multicolumn{2}{|c|}{ Baseline } & \multirow{2}{*}{$\begin{array}{l}\begin{array}{l}\text { Baseline } \\
\text { difference }\end{array} \\
P \text {-value }\end{array}$} & \multicolumn{2}{|c|}{12 weeks } & \multicolumn{2}{|c|}{ Change } & \multicolumn{3}{|c|}{$\begin{array}{l}\text { Mean difference between } \\
\text { groups (garlic vs placebo) }\end{array}$} \\
\hline \multirow[t]{2}{*}{$\begin{array}{l}\text { Office } \\
\text { BP }\end{array}$} & Variable & Details & & & Mean & SD & & Mean & SD & Mean & SD & $\begin{array}{l}\text { Mean } \\
\text { difference }\end{array}$ & SE & $P$-value \\
\hline & \multirow[t]{2}{*}{ High AP, female } & $\mathrm{mmHg}$ & Placebo & 11 & 17.5 & 6.2 & & 19.5 & 11.5 & -1.4 & 5.8 & & & \\
\hline & & & Garlic & 14 & 19 & 4.6 & NS & 18.4 & 6.4 & -3.7 & 5.4 & -2.3 & 2.3 & 0.32 \\
\hline & \multirow[t]{2}{*}{ Low AP } & $\mathrm{mmHg}$ & Placebo & 24 & 6.9 & 3.5 & & 8.7 & 4.6 & 1.8 & 4.6 & & & \\
\hline & & & Garlic & 26 & 6.6 & 2.8 & NS & 8.5 & 4.1 & 1.8 & 4.2 & -0.03 & 1.3 & 0.98 \\
\hline & \multirow[t]{2}{*}{ RM } & $\%$ & Placebo & 38 & 65.1 & 6.6 & & 64.3 & 5.8 & -0.82 & 5.2 & & & \\
\hline & & & Garlic & 49 & 63.6 & 8.2 & NS & 64.3 & 5.7 & 0.67 & 6.5 & -1.5 & 1.3 & 0.25 \\
\hline & \multirow[t]{2}{*}{$\mathrm{Al}$} & $\%$ & Placebo & 35 & 21.7 & 11.6 & & 21.8 & 10 & 0.82 & 9.8 & & & \\
\hline & & & Garlic & 48 & 24 & 13.8 & NS & 24.2 & II.I & -0.15 & 9.4 & -0.97 & 3.1 & NS \\
\hline & \multirow[t]{2}{*}{ Al high* } & $\%$ & Placebo & 12 & 31.8 & 7.2 & & 26.4 & 8.3 & -5 & 10.5 & & & \\
\hline & & & Garlic & 22 & 36.2 & 7.7 & NS & 31.3 & 8.9 & -4.6 & 7.9 & 1.5 & 3.4 & 0.66 \\
\hline & \multirow[t]{2}{*}{ Al low } & $\%$ & Placebo & 23 & 16.4 & 9.7 & & 19.3 & 10.1 & 4.3 & 7.6 & & & \\
\hline & & & Garlic & 25 & 13.8 & 8.3 & NS & 18.1 & 9 & 3.6 & 9.1 & -0.5 & 2.5 & 0.85 \\
\hline & \multirow[t]{2}{*}{ PWV } & $\mathrm{m} / \mathrm{s}$ & Placebo & 38 & 9.3 & 1.8 & & 9.2 & 1.8 & -0.071 & 0.33 & & & \\
\hline & & & Garlic & 49 & 9.8 & 1.9 & NS & 9.5 & 1.6 & -0.28 & 0.58 & -0.16 & 0.11 & 0.13 \\
\hline & \multirow[t]{2}{*}{ PWV high" } & $\mathrm{m} / \mathrm{s}$ & Placebo & 25 & 9.6 & 1.9 & & 9.5 & 2 & -0.15 & 0.35 & & & \\
\hline & & & Garlic & 31 & 10.2 & 2.2 & NS & 9.7 & 1.9 & -0.49 & 0.62 & -0.33 & 0.14 & 0.02 \\
\hline & \multirow[t]{2}{*}{ PWV normal } & $\mathrm{m} / \mathrm{s}$ & Placebo & 13 & 8.5 & 1.2 & & 8.6 & 1.2 & 0.08 & 0.25 & & & \\
\hline & & & Garlic & 18 & 9 & 0.8 & NS & 9.1 & 0.9 & 0.07 & 0.29 & 0.01 & 0.1 & 0.89 \\
\hline
\end{tabular}

Notes: *Upper 50th percentile of population range adjusted for age and sex; ${ }^{19}{ }^{*}$ above normal population range, adjusted for age and sex. ${ }^{20}$ High AP/Al, upper $50 \%$ of population average; low AP/AI, lower $50 \%$ of population average; cardiac index, cardiac output from left ventricle in I minute per body surface area.

Abbreviations: $\mathrm{Al}$, augmentation index (at 75\%); AP, augmentation pressure; BP, blood pressure; $\mathrm{CSBP}$, central systolic BP; cDBP, central diastolic BP; cPP, central pulse pressure; HR, heart rate; MAP, mean arterial pressure; NS, not significant; PWV, pulse-wave velocity; RM, reflection magnitude; SD, standard deviation; SE, standard error; TVR, total vascular resistance.

A

\section{SBP change}

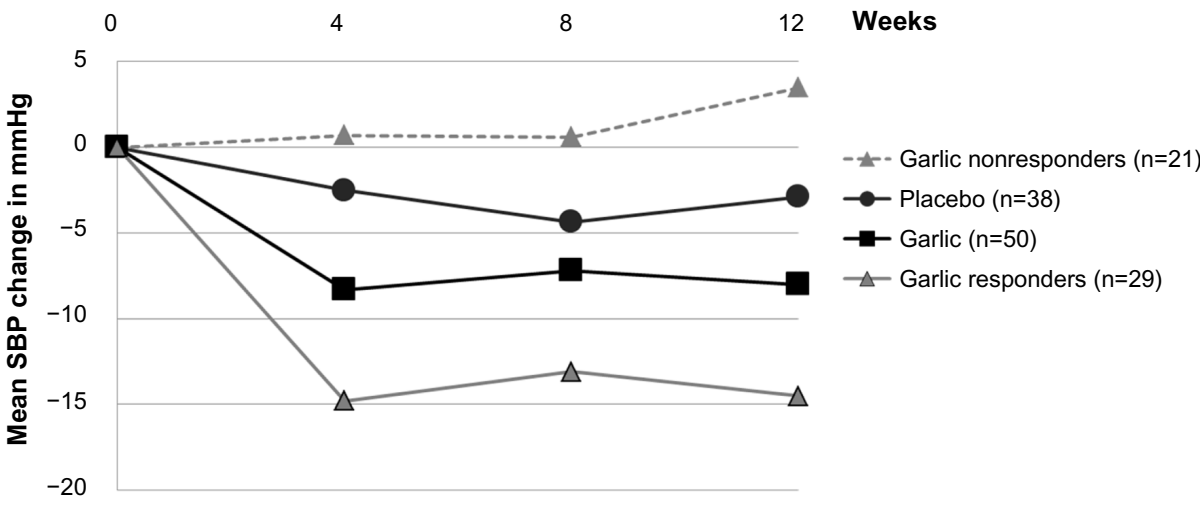

B

DBP change

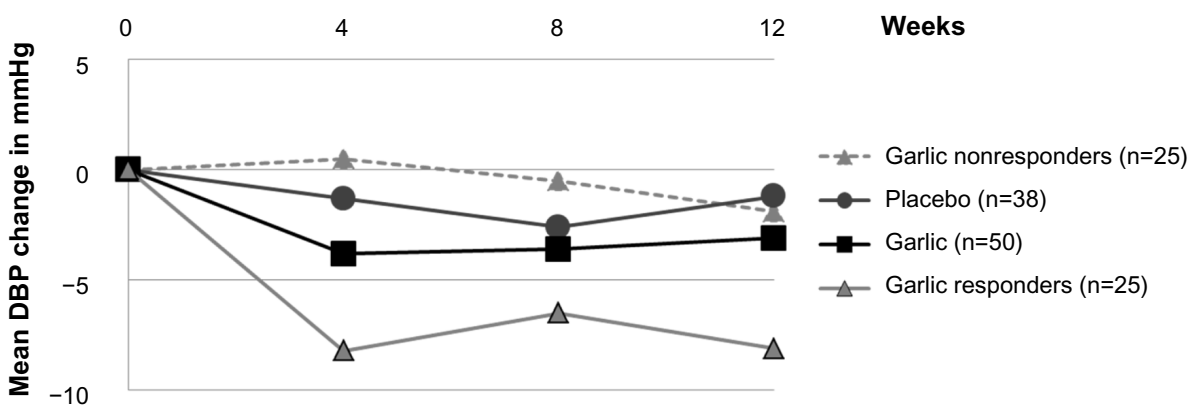

Figure 2 Effect of aged garlic extract on blood pressure.

Notes: Mean change in SBP (A) and DBP (B) over 12 weeks in the placebo and garlic groups, and responder (SBP change $\geq 5 \mathrm{mmHg}$, DBP change $\geq 3 \mathrm{mmHg}$ ) and nonresponder subgroups.

Abbreviations: SBP, systolic blood pressure; DBP, diastolic BP. 
Table 3 Cardiovascular markers by blood test

\begin{tabular}{|c|c|c|c|c|c|c|c|c|c|c|c|c|}
\hline \multirow[t]{2}{*}{ Blood tests } & \multirow[t]{2}{*}{ Reference range } & \multirow[t]{2}{*}{ Units } & \multirow[t]{2}{*}{ Group } & \multirow[t]{2}{*}{$\mathbf{n}$} & \multicolumn{2}{|c|}{ Baseline } & \multirow{2}{*}{$\begin{array}{l}\text { Baseline } \\
\text { difference }\end{array}$} & \multicolumn{2}{|c|}{12 weeks } & \multicolumn{2}{|c|}{$\begin{array}{l}\text { Change from } \\
\text { baseline }\end{array}$} & \multirow{2}{*}{$\begin{array}{l}\text { Change } \\
\text { difference } \\
P \text {-value }\end{array}$} \\
\hline & & & & & Mean & SD & & Mean & SD & Mean & SD & \\
\hline \multicolumn{13}{|l|}{ Lipids } \\
\hline \multirow[t]{2}{*}{ TG } & & $\mathrm{mmol} / \mathrm{L}$ & Placebo & 38 & 1.6 & 0.9 & NS & 1.5 & 1 & -0.07 & 0.66 & 0.07 \\
\hline & & & Garlic & 50 & 1.2 & 0.6 & & 1.3 & 0.8 & 0.15 & 0.45 & \\
\hline \multirow[t]{2}{*}{ TC } & $<5.5$ & $\mathrm{mmol} / \mathrm{L}$ & Placebo & 38 & 5.3 & 1 & NS & 5.3 & 1.3 & 0.003 & 0.84 & NS \\
\hline & & & Garlic & 50 & 5.5 & 1.3 & & 5.5 & 1.2 & -0.01 & 0.75 & \\
\hline TC subgroups & High & & Placebo & 17 & 6.1 & 0.6 & NS & 5.9 & 1.5 & -0.16 & I.I & NS \\
\hline & & & Garlic & 23 & 6.6 & 1 & & 6.3 & 0.9 & -0.27 & 0.79 & \\
\hline & Normal & & Placebo & 21 & 4.6 & 0.6 & NS & 4.7 & 0.7 & 0.13 & 0.54 & NS \\
\hline & & & Garlic & 27 & 4.6 & 0.7 & & 4.8 & 0.9 & 0.21 & 0.64 & \\
\hline LDL & $<3.4$ & $\mathrm{mmol} / \mathrm{L}$ & Placebo & 37 & 3.1 & 0.9 & NS & 3.1 & 1 & -0.028 & 0.52 & NS \\
\hline & & & Garlic & 50 & 0.8 & I.I & & 3.2 & 0.9 & -0.12 & 0.7 & \\
\hline LDL subgroups & High & & Placebo & 13 & 4 & 0.6 & & 3.8 & 1.2 & -0.2 & 0.76 & NS \\
\hline & & & Garlic & 18 & 4.4 & 1 & NS & 4 & 0.6 & -0.46 & 0.91 & \\
\hline & Normal & & Placebo & 24 & 2.6 & 0.6 & & 2.7 & 0.6 & 0.061 & 0.29 & NS \\
\hline & & & Garlic & 32 & 2.7 & 0.6 & NS & 276 & 0.7 & 0.069 & 0.46 & \\
\hline HDL & $0.9-2.2$ & $\mathrm{mmol} / \mathrm{L}$ & Placebo & 38 & 1.5 & 0.6 & NS & 1.4 & 0.5 & -0.068 & 0.39 & NS \\
\hline & & & Garlic & 50 & 1.7 & 0.6 & & 1.7 & 0.6 & -0.02 & 0.27 & \\
\hline $\mathrm{TC} / \mathrm{HDL}$ & & & Placebo & 38 & 3.9 & 1.2 & NS & 3.9 & 1.1 & -0.0026 & 0.48 & NS \\
\hline & & & Garlic & 50 & 3.5 & $\mathrm{I} . \mathrm{I}$ & & 3.5 & I & -0.0034 & 0.47 & \\
\hline ApoA & $105-185$ & $\mathrm{mg} / \mathrm{dL}$ & Placebo & 38 & I70.I & 34.8 & 0.06 & 169.2 & 33.4 & -0.92 & 21 & NS \\
\hline & & & Garlic & 50 & 184.9 & 38 & & 187.7 & 41.5 & 2.78 & 28.27 & \\
\hline ApoA subgroups & High & & Placebo & 8 & 219 & 34 & NS & 209.8 & 35.4 & -9.25 & 27.28 & NS \\
\hline & & & Garlic & 24 & 217 & 23.1 & & 210.7 & 29.2 & -6.25 & 23.81 & \\
\hline & Normal & & Placebo & 30 & I57.I & 20.9 & NS & 158.4 & 23.3 & 1.3 & 18.94 & NS \\
\hline & & & Garlic & 25 & 154.9 & 21.5 & & 164.7 & 40 & 9.76 & 29.69 & \\
\hline ApoB & $55-125$ & $\mathrm{mg} / \mathrm{dL}$ & Placebo & 38 & 101.5 & 25.5 & NS & 101.2 & 31 & -0.26 & 16.38 & NS \\
\hline & & & Garlic & 50 & 102 & 32.5 & & 100.7 & 30.8 & -1.34 & 28.4 & \\
\hline ApoB subgroups & High & & Placebo & 5 & 146.2 & 23.9 & NS & 148.2 & 51.1 & 2 & 32.57 & NS \\
\hline & & & Garlic & 7 & 160.9 & 32.2 & & 129.7 & 57.4 & -31.14 & 64.34 & \\
\hline & Normal & & Placebo & 32 & 96.1 & 16.4 & NS & 94.9 & 19.4 & -1.15 & $13.1 \mid$ & NS \\
\hline & & & Garlic & 42 & 92.4 & 20.6 & & 95.5 & 21.8 & 3.14 & 13.17 & \\
\hline Homocysteine & $3.7-13.9$ & $\mu \mathrm{mol} / \mathrm{L}$ & Placebo & 37 & 14.1 & 3.4 & NS & 13.5 & 4.3 & -0.67 & 4.19 & NS \\
\hline & & & Garlic & 50 & 14.2 & 3.7 & & 14.9 & 4.5 & 0.67 & 3.88 & \\
\hline Homocysteine & High & & Placebo & 17 & 17 & 2.8 & NS & 15 & 5.4 & -1.97 & 5.34 & NS \\
\hline & & & Garlic & 26 & 17 & 2.6 & & 16.9 & 4.6 & -0.12 & 4.15 & \\
\hline & Normal & & Placebo & 20 & 11.8 & 1.3 & NS & 12.2 & 2.6 & 0.43 & 2.55 & NS \\
\hline & & & Garlic & 24 & 11.2 & 1.7 & & 12.8 & 3.2 & 1.57 & 3.44 & \\
\hline Platelet functio & & & & & & & & & & & & \\
\hline Epi/Coll & $120-150$ & s & Placebo & 35 & 143.3 & 45.8 & NS & 142.7 & 55.4 & -0.52 & 37.7 & NS \\
\hline & & & Garlic & 46 & 142.3 & 47.2 & & 138.6 & 54.5 & -3.67 & 45.2 & \\
\hline Epi subgroups & Thick under 120 & & Placebo & 12 & 101.7 & 15.7 & NS & 110.1 & 25.6 & 8.42 & 21.63 & NS \\
\hline & & & Garlic & 18 & 102.5 & 10.4 & & 107.9 & 32.7 & 5.4 & 32.9 & \\
\hline & Thin over 150 & & Placebo & 13 & 188.8 & 40.4 & NS & 178.8 & 73 & -10 & 56.7 & NS \\
\hline & & & Garlic & 16 & 193.4 & 41.9 & & 180 & 61.9 & -13.34 & 63.5 & \\
\hline ADP/Coll & $85-110$ & s & Placebo & 34 & 95.4 & 21.6 & NS & 95.9 & 26.1 & 0.47 & 17.25 & NS \\
\hline & & & Garlic & 41 & 92.8 & 21 & & 89.6 & 16.3 & -3.12 & 14.2 & \\
\hline & Thick under 85 & & Placebo & 12 & 75.4 & 5.9 & NS & 79.6 & 12.4 & 4.17 & 10.9 & NS \\
\hline & & & Garlic & 17 & 77.4 & 4.6 & & 84.4 & 12.1 & 7.06 & 11.1 & \\
\hline & Thin over 110 & & Placebo & 6 & 132 & 15.1 & NS & 125.7 & 41.4 & -6.33 & 26.8 & NS \\
\hline & & & Garlic & 5 & 137.4 & 25 & & 117 & 19.3 & -20.4 & 10 & \\
\hline Inflammatory & larkers & & & & & & & & & & & \\
\hline CRP & & $\mathrm{mg} / \mathrm{L}$ & Placebo & 38 & 3.2 & 4.6 & NS & 4 & 4.6 & 0.8 & 3.7 & NS \\
\hline & & & Garlic & 50 & 2.7 & 3.7 & & 3.1 & 4.2 & 0.4 & 3.8 & \\
\hline $\mathrm{TNF} \alpha$ & & $\mathrm{fg} / \mathrm{mL}$ & Placebo & 27 & 245.6 & 269.5 & NS & 448.7 & 550.8 & 203.1 & 475.9 & 0.05 \\
\hline & & & Garlic & 31 & 238.9 & 310.6 & & 227.8 & 196.6 & -11.1 & 358.6 & \\
\hline IL-I $\beta$ & & $\mathrm{fg} / \mathrm{mL}$ & Placebo & 27 & 145.6 & 89.2 & NS & 139.8 & 104.2 & -5.8 & 96.0 & NS \\
\hline & & & Garlic & 31 & 211.7 & 439.0 & & 114.3 & 48.3 & -97.4 & 433.6 & \\
\hline
\end{tabular}

Abbreviations: ADP, adenosine diphosphate; ApoA, Apolipoprotein A; ApoB, Apolipoprotein B; Coll, collagen; Epi, epinephrine; HDL, high-density lipoprotein; LDL, lowdensity lipoprotein; NS, not significant; SD, standard deviation; TC/HDL, total cholesterol:HDL ratio; TG, triglycerides. 
Table 4 Kidney- and liver-function test results

\begin{tabular}{|c|c|c|c|c|c|c|c|c|c|c|c|c|}
\hline \multirow[t]{2}{*}{ Blood tests } & \multirow[t]{2}{*}{$\begin{array}{l}\text { Reference } \\
\text { range }\end{array}$} & \multirow[t]{2}{*}{ Unit } & \multirow[t]{2}{*}{ Group } & \multirow[t]{2}{*}{$\mathbf{n}$} & \multicolumn{2}{|c|}{ Baseline } & \multirow{2}{*}{$\begin{array}{l}\text { Baseline } \\
\text { difference } \\
P \text {-value }\end{array}$} & \multicolumn{2}{|c|}{12 weeks } & \multicolumn{2}{|c|}{$\begin{array}{l}\text { Change from } \\
\text { baseline }\end{array}$} & \multirow{2}{*}{$\begin{array}{l}\text { Change } \\
\text { difference } \\
P \text {-value }\end{array}$} \\
\hline & & & & & Mean & SD & & Mean & SD & Mean & SD & \\
\hline \multicolumn{13}{|c|}{ Kidney function } \\
\hline \multirow[t]{2}{*}{$\mathrm{Na}$} & & $\mathrm{mmol} / \mathrm{L}$ & Placebo & 38 & 139.8 & 2.7 & NS & 139.8 & 2.2 & 0.1 & 1.9 & NS \\
\hline & & & Garlic & 49 & 140.1 & 2.3 & & 139.9 & 2.3 & -0.2 & 2.3 & \\
\hline \multirow[t]{2}{*}{ K } & & $\mathrm{mmol} / \mathrm{L}$ & Placebo & 38 & 4.46 & 0.53 & NS & 4.3 & 0.4 & -0.1 & 0.6 & NS \\
\hline & & & Garlic & 49 & 4.55 & 0.47 & & 4.4 & 0.4 & -0.1 & 0.5 & \\
\hline \multirow[t]{2}{*}{$\mathrm{Cl}$} & & $\mathrm{mmol} / \mathrm{L}$ & Placebo & 38 & 103.1 & 3.4 & NS & 103.3 & 2.9 & 0.3 & 2.8 & NS \\
\hline & & & Garlic & 49 & 103.5 & 2.9 & & 103.3 & 2.6 & -0.1 & 2.5 & \\
\hline \multirow[t]{2}{*}{ Bicarbonate } & & $\mathrm{mmol} / \mathrm{L}$ & Placebo & 38 & 28.3 & 2.8 & NS & 30.1 & 2.2 & 1.8 & 3.1 & 0.03 \\
\hline & & & Garlic & 49 & 29.1 & 2.6 & & 29.5 & 2.4 & 0.3 & 2.9 & \\
\hline \multirow[t]{2}{*}{ Urea } & & $\mathrm{mmol} / \mathrm{L}$ & Placebo & 38 & 6.1 & 1.8 & NS & 6.2 & 1.6 & 0.1 & 1.5 & NS \\
\hline & & & Garlic & 49 & 5.8 & 1.4 & & 5.7 & 1.4 & 0.1 & 1.4 & \\
\hline \multirow[t]{2}{*}{ eGFR } & & $\mathrm{mL} / \mathrm{min}$ & Placebo & 38 & 86.2 & 9.4 & NS & 84.9 & 9.6 & -1.2 & 5.1 & 0.06 \\
\hline & & & Garlic & 8 & 85.6 & 9.5 & & 86.4 & 9.3 & 0.9 & 4.8 & \\
\hline \multirow[t]{2}{*}{ Creatinine } & & $\mu \mathrm{mol} / \mathrm{L}$ & Placebo & 38 & 69.7 & 18.5 & NS & 70.63 & 17.34 & I & 7.8 & 0.05 \\
\hline & & & Garlic & 49 & 69.8 & 15.4 & & 67.18 & 16.42 & -2.8 & 9.1 & \\
\hline \multirow[t]{2}{*}{$\mathrm{Ca}$} & & $\mathrm{mmol} / \mathrm{L}$ & Placebo & 38 & 2.3 & 0.1 & NS & 2.33 & 0.096 & -0.01 & 0.1 & NS \\
\hline & & & Garlic & 50 & 2.3 & 0.1 & & 2.33 & 0.07 & -0.0012 & 0.1 & \\
\hline \multirow[t]{2}{*}{ CorCa } & & $\mathrm{mmol} / \mathrm{L}$ & Placebo & 36 & 2.3 & 0.1 & NS & 2.23 & 0.07 & -0.02 & 0.1 & NS \\
\hline & & & Garlic & 50 & 2.3 & 0.1 & & 2.24 & 0.07 & -0.004 & 0.1 & \\
\hline \multirow[t]{2}{*}{ Phosphate } & & $\mathrm{mmol} / \mathrm{L}$ & Placebo & 38 & 1.06 & 0.2 & NS & 1.02 & 0.2 & -0.4 & 0.2 & NS \\
\hline & & & Garlic & 50 & 1.04 & 0.2 & & 1.05 & 0.1 & 0.01 & 0.2 & \\
\hline Urate & & $\mathrm{mmol} / \mathrm{L}$ & Placebo & 38 & 0.37 & 0.1 & NS & 0.36 & 0.096 & -0.01 & 0.06 & \\
\hline & & & Garlic & 50 & 0.35 & 0.1 & & 0.36 & 0.09 & -0.01 & 0.06 & NS \\
\hline Liver functio & & & & & & & & & & & & \\
\hline Bilirubin & & $\mu \mathrm{mol} / \mathrm{L}$ & Placebo & 38 & 11.4 & 4.7 & NS & 12 & 4.22 & 0.6 & 3.5 & NS \\
\hline & & & Garlic & 50 & 11.4 & 4.5 & & 11.7 & 4.4 & 0.3 & 3.4 & \\
\hline ALT & & $U / L$ & Placebo & 38 & 30.5 & 14.1 & 0.12 & 30.8 & 14.5 & 0.3 & 10.1 & NS \\
\hline & & & Garlic & 50 & 25.7 & 13.8 & & 25.5 & 14.1 & -0.3 & 6.1 & \\
\hline AST & & $\mathrm{U} / \mathrm{L}$ & Placebo & 38 & 26.2 & 6.8 & NS & 28.3 & 10.7 & 2.1 & 7.7 & 0.03 \\
\hline & & & Garlic & 50 & 26.5 & 8.1 & & 25.5 & 7.3 & -1 & 5.7 & \\
\hline ALP & & $\mathrm{U} / \mathrm{L}$ & Placebo & 38 & 67.4 & 14.4 & 0.005 & 71.2 & 18.1 & 3.8 & 10.9 & NS \\
\hline & & & Garlic & 50 & 78.6 & 22 & & 80.1 & 24.4 & 1.5 & 10.8 & \\
\hline GGT & & $U / L$ & Placebo & 38 & 39.2 & 58 & 0.5 & 42.9 & 90.1 & 3.8 & 35.5 & NS \\
\hline & & & Garlic & 50 & 32.7 & 30.3 & & 34.8 & 34.8 & 2.1 & 12.2 & \\
\hline Total protein & & $g / L$ & Placebo & 38 & 72.6 & 4.1 & NS & 73 & 4.3 & 0.3 & 3.8 & NS \\
\hline & & & Garlic & 50 & 72.7 & 5.1 & & 73 & 3.7 & 0.3 & 5.1 & \\
\hline Albumin & & $g / L$ & Placebo & 38 & 44.3 & 3.3 & NS & 45 & 3.5 & 0.7 & 2.7 & NS \\
\hline & & & Garlic & 50 & 44.2 & 2.9 & & 44.4 & 2.5 & 0.2 & 3.1 & \\
\hline Globulin & & $g / L$ & Placebo & 38 & 28.3 & 3 & NS & 28 & 3.3 & -0.3 & 2.1 & NS \\
\hline & & & Garlic & 50 & 28.5 & 3.4 & & 28.5 & 3.1 & 0.1 & 2.5 & \\
\hline Glucose & & $\mathrm{mmol} / \mathrm{L}$ & Placebo & 38 & 5.4 & I.I & & 5.3 & 1 & -0.1 & 0.5 & 0.19 \\
\hline & & & Garlic & 49 & 5.3 & 1.2 & NS & 5.5 & 1.7 & 0.2 & 1.1 & \\
\hline
\end{tabular}

Abbreviations: CorCa, corrected calcium for albumin; eGFR, estimated glomerular filtration rate; NS, not significant; SD, standard deviation.

to take two trial capsules a day for 12 weeks, and about $80 \%$ stated they would be willing to continue taking the capsules if they helped with BP control (Table 5). Blinding success was assessed at the end of the trial by questionnaire. While more participants in the garlic group (42\%) than in the placebo group (21\%) guessed their allocation correctly, a larger proportion in both groups were unsure or incorrect (garlic 58\%, placebo $79 \% ; P=0.023$; Table 5).

\section{Discussion}

Our trial suggests that aged garlic extract is superior to placebo in lowering BP in patients with uncontrolled hypertension. A dosage of two capsules daily containing $1.2 \mathrm{~g}$ of aged garlic extract and $1.2 \mathrm{mg}$ of SAC significantly lowered SBP by $5 \mathrm{mmHg}$ compared with placebo over 12 weeks $(P=0.016)$, and was highly tolerated. BP changes were observed only in a proportion of participants, whereby 50\%-58\% responded 
Table 5 Tolerability, acceptability, and blinding

\begin{tabular}{|c|c|c|c|}
\hline \multirow[t]{2}{*}{ Tolerability } & $\begin{array}{l}\text { All } \\
(n=88)\end{array}$ & $\begin{array}{l}\text { Garlic } \\
(n=50)\end{array}$ & $\begin{array}{l}\text { Placebo } \\
(n=38)\end{array}$ \\
\hline & n (\%) & $\overline{n(\%)}$ & $\mathrm{n}(\%)$ \\
\hline \multicolumn{4}{|l|}{ Side effects } \\
\hline Total & $28(32)$ & $18(36)$ & $10(26)$ \\
\hline Reflux & $7(8)$ & $5(10)$ & $2(5)$ \\
\hline Improved digestion & $6(7)$ & $5(10)$ & $I(2)$ \\
\hline Garlic taste & $3(3)$ & $3(6)$ & 0 \\
\hline Hot flushes & $4(5)$ & $3(6)$ & $I(2)$ \\
\hline Burping & $4(5)$ & $2(4)$ & $2(5)$ \\
\hline Bloating & $3(3)$ & $I(2)$ & $2(5)$ \\
\hline Flatulence & $3(3)$ & $I(2)$ & $2(5)$ \\
\hline Dizzy & $I(I)$ & $I(2)$ & $0(0)$ \\
\hline Nosebleed & $I(I)$ & I (2) & 0 \\
\hline Diarrhea & $2(2)$ & I (2) & I (2) \\
\hline Indigestion & $2(2)$ & $I(2)$ & $I(2)$ \\
\hline Difficulty swallowing capsules & $2(2)$ & I (2) & I (2) \\
\hline Nausea & $I(I)$ & 0 & $I(2)$ \\
\hline Dry mouth & $I(I)$ & 0 & I (2) \\
\hline \multicolumn{4}{|l|}{ Acceptability } \\
\hline \multicolumn{4}{|l|}{ Easy taking capsules } \\
\hline Easy & $84(95)$ & $48(96)$ & $36(95)$ \\
\hline \multicolumn{4}{|l|}{ Acceptable } \\
\hline Acceptable & $88(100)$ & $50(100)$ & $38(100)$ \\
\hline \multicolumn{4}{|l|}{ Willing to continue } \\
\hline Agree & $74(84)$ & $44(88)$ & $30(79)$ \\
\hline Unsure & $11(13)$ & $4(8)$ & $7(18)$ \\
\hline Disagree & $3(3)$ & $2(4)$ & $\mathrm{I}(3)$ \\
\hline \multicolumn{4}{|c|}{ Willing to spend (AU\$0.3/capsule) } \\
\hline Agree & $70(80)$ & $42(84)$ & $28(76)$ \\
\hline Unsure & $14(16)$ & $7(14)$ & $7(19)$ \\
\hline Disagree & $3(3)$ & $\mathrm{I}(2)$ & $2(5)$ \\
\hline \multicolumn{4}{|l|}{ Blinding } \\
\hline Correct & $29(33)$ & $21(42)$ & $8(21)$ \\
\hline Unsure & $46(52)$ & $22(44)$ & $24(63)$ \\
\hline Incorrect & $13(15)$ & $7(14)$ & $6(16)$ \\
\hline
\end{tabular}

Notes: Acceptability was measured using 5-point Likert scales. The two highest categories were combined (eg, very easy/easy, very acceptable/acceptable, strongly agree/agree)

to treatment by a reduction in SBP of more than $5 \mathrm{mmHg}$ $(58 \%)$ or a reduction in DBP of more than $3 \mathrm{mmHg}(50 \%)$. Subgroup analysis of responders revealed a significant average reduction of $11 \mathrm{mmHg} \mathrm{SBP}$ and $6 \mathrm{mmHg}$ DBP compared to placebo $(P<0.001)$.

Interindividual differences in BP response to garlic treatment are biologically plausible ${ }^{9}$ and in line with our previous research, whereby SBP was reduced significantly in about $70 \%$ of participants. ${ }^{6}$ Average BP reduction of responders in this trial is also consistent with meta-analyses of the effect of garlic on BP in hypertensives by $9-10 \mathrm{mmHg}$ SBP and by $4-8 \mathrm{mmHg}$ DBP $(P<0.001){ }^{8,23}$

Garlic-derived polysulfides can influence BP via the nitric oxide- and hydrogen sulfide-signaling pathways. ${ }^{9}$ Several dietary and genetic factors influence the efficiency of these pathways, including vitamin $\mathrm{B}_{6}$, vitamin $\mathrm{B}_{12}$, and folate levels or genetic polymorphisms, eg, the transsulfuration gene $C B S$, and hence support or ameliorate the BP response to garlic intake. ${ }^{9}$

In our study, responders and nonresponders did not differ by age, sex, body mass index, or BP-medication intake. Therefore, we speculate that the variability in BP response to garlic in this study may be linked to underlying vitamin $\mathrm{B}_{6}$, vitamin $\mathrm{B}_{12}$ or folate deficiency, or genetic polymorphisms.

Variation in vitamin $\mathrm{B}_{12}$ levels prevalent in middle-aged populations is supported by the relatively high mean homocysteine levels found in our study sample, and the lack of effect of garlic on homocysteine levels, in line with the literature linking low vitamin $\mathrm{B}_{12}$ levels and high homocysteine levels with hypertension and cardiovascular disease. ${ }^{24}$

Secondary outcome measures, including central hemodynamics, such as central SBP, central pulse pressure, mean arterial pressure, total vascular resistance, arterial stiffness, and PWV, were positively influenced by aged garlic extract. Central hemodynamic measures are regarded as more important predictors than peripheral BP for cardiovascular disease. ${ }^{11}$ Our findings are in line with a previous trial showing a beneficial effect of aged garlic extract plus coenzyme $Q_{10}$ on $P W V$ and endothelial function, ${ }^{25}$ and provide new evidence that aged garlic extract has the potential to reduce central BP and arterial stiffness in individuals with uncontrolled hypertension.

While we did not find significant differences in other cardiovascular markers, favorable effects observed in the inflammatory marker TNF $\alpha$, as well as total cholesterol, LDL cholesterol, and correlated ApoA and ApoB levels, were in line with previous research, whereby garlic was found to reduce the proinflammatory markers IL-1 $\beta$ and $\mathrm{TNF} \alpha$, subsequent activation of nuclear factor $\mathrm{NF} \kappa \mathrm{B}$, and levels of oxidative LDL in vitro. ${ }^{26,27}$ Furthermore, a previous meta-analysis of 39 trials suggested garlic was effective in reducing total cholesterol and LDL cholesterol by $10 \%$ in individuals with slightly elevated levels. ${ }^{28}$

Platelet-function testing provided evidence that Kyolic aged garlic extract was safe, even if taken in addition to blood-thinning medication, which is in line with previous research, ${ }^{10}$ and in contrast to raw garlic intake. ${ }^{29}$ In our sample, Kyolic aged garlic extract tended to normalize platelet function.

While our study was adequately powered and adjusted for confounding factors for the primary outcome measures - SBP and DBP - allowing also for exploration by subgroups of responders/nonresponders, our study has a few limitations. 
The dropout rate in the placebo group (22\%) was higher than in the garlic group (9\%), which may have led to some biased results. Assessments of the effect of aged garlic extract on most secondary outcome measures, including arterial stiffness measures and cardiovascular blood markers, were limited by the smaller sample size in subgroups of participants with elevated levels of these markers at baseline. Intake of prescription medication, including medicines for BP, lipidlowering agents, or hormone-replacement therapy were not matched between the groups, and numbers were too small in each category to undertake subgroup analyses. However, differences between the groups were not statistically significant, and did not change results appreciably when incorporated in the analyses as covariates.

Further larger studies are needed to assess the full potential of aged garlic extract on arterial stiffness measures, including PWV and augmentation pressure, as well as on cardiovascular biomarkers, such as inflammatory markers, lipids, and platelet function. Additionally, future research should test the hypothesis that individuals' responsiveness to aged garlic extract intake may be dependent on underlying dietary and genetic factors, such as vitamin $\mathrm{B}_{6}$, vitamin $\mathrm{B}_{12}$, folate levels, and $C B S$ gene polymorphisms.

\section{Acknowledgments}

We thank all patients, general practices, doctors, and staff for their participation in the trial. We are grateful to the research nurse Adela Cretoiu, who was instrumental in liaising with practices and patients. This trial was supported by a grant from Wakunaga of America Co Ltd, who supplied trial capsules and provided funding for costs of tests and research assistance. Wakunaga of America was not involved in study design, data collection, analysis, or preparation of the manuscript.

\section{Author contributions}

KR and AS conceptualized the study, and KR acquired funding and oversaw data collection by NT. KR and NT undertook data analysis. All authors contributed toward drafting and critically revising the paper and agree to be accountable for all aspects of the work.

\section{Disclosure}

The authors report no conflicts of interest in this work.

\section{References}

1. World Heart Federation. Cardiovascular disease risk factors. 2012. Available from: http://www.world-heart-federation.org/press/fact-sheets/ cardiovascular-disease-risk-factors. Accessed December 9, 2015.
2. Lawes CM, Vander Hoorn S, Rodgers A. Global burden of bloodpressure-related disease, 2001. Lancet. 2008;371(9623):1513-1518.

3. Martiniuk AL, Lee CM, Lawes CM, et al. Hypertension: its prevalence and population-attributable fraction for mortality from cardiovascular disease in the Asia-Pacific region. $J$ Hypertens. 2007;25(1): 73-79.

4. Briganti EM, Shaw JE, Chadban SJ, et al. Untreated hypertension among Australian adults: the 1999-2000 Australian Diabetes, Obesity and Lifestyle study (AusDiab). Med J Aust. 2003;179(3): 135-139.

5. Ried K, Frank OR, Stocks NP. Aged garlic extract lowers blood pressure in patients with treated but uncontrolled hypertension: a randomised controlled trial. Maturitas. 2010;67(2):144-150.

6. Ried K, Frank OR, Stocks NP. Aged garlic extract reduces blood pressure in hypertensives: a dose-response trial. Eur J Clin Nutr. 2013;67(1):64-70.

7. Ried K, Frank OR, Stocks NP, Fakler P, Sullivan T. Effect of garlic on blood pressure: a systematic review and meta-analysis. BMC Cardiovasc Disord. 2008;8:13.

8. Ried K. Garlic lowers blood pressure in hypertensive individuals, regulates serum cholesterol, and stimulates immunity: an updated meta-analysis and review. $J$ Nutr. 2016;146:1S-8S. In pres.

9. Ried K, Fakler P. Potential of garlic (Allium sativum) in lowering high blood pressure: mechanisms of action and clinical relevance. Integrat Blood Press Control. 2014;7:71-82.

10. Macan H, Uykimpang R, Alconcel M, et al. Aged garlic extract may be safe for patients on warfarin therapy. J Nutr. 2006;136 (3 Suppl):793S-795S

11. Laurent S, Cockcroft J, Van Bortel L, et al. Expert consensus document on arterial stiffness: methodological issues and clinical applications. Eur Heart J. 2006;27(21):2588-2605.

12. O'Rourke M. Arterial stiffness, systolic blood pressure, and logical treatment of arterial hypertension. Hypertension. 1990;15(4):339-347.

13. Wakunaga. Kyolic Reserve. Available from: http://www.kyolic.com/ product/category/reserve. Accessed December 9, 2015.

14. Amagase H. Clarifying the real bioactive constituents of garlic. J Nutr. 2006;136(3 Suppl):716S-725S.

15. Wassertheurer S, Kropf J, Weber T, et al. A new oscillometric method for pulse wave analysis: comparison with a common tonometric method. J Hum Hypertens. 2010;24(8):498-504.

16. Weber T, Wassertheurer S, Rammer M, et al. Validation of a brachial cuff-based method for estimating central systolic blood pressure. Hypertension. 2011;58(5):825-832.

17. Weiss W, Gohlisch C, Harsch-Gladisch C, Tölle M, Zidek W, van der Giet M. Oscillometric estimation of central blood pressure: validation of the Mobil-O-Graph in comparison with the SphygmoCor device. Blood Press Monit. 2012;17(3):128-131.

18. IEM. Mobil-O-Graph 24h PWA. Available from: http://www.iem.de/ en/products/mobil-o-graph.html. Accessed December 9, 2015.

19. Nunan D, Wassertheurer S, Lasserson D, et al. Assessment of central haemomodynamics from a brachial cuff in a community setting. $B M C$ Cardiovasc Disord. 2012;12:48.

20. Nunan D, Fleming S, Hametner B, Wassertheurer S. Performance of pulse wave velocity measured using a brachial cuff in a community setting. Blood Press Monit. 2014;19(6):315-319.

21. Bulpitt C. Responders and non-responders to antihypertensive treatment. Drugs. 1988;35(6):142-146.

22. Cohen S, Kamarck T, Mermelstein R. A global measure of perceived stress. J Health Soc Behav. 1983;24(4):385-396.

23. Rohner A, Ried K, Sobenin IA, Bucher HC, Nordmann AJ. A systematic review and metaanalysis on the effects of garlic preparations on blood pressure in individuals with hypertension. Am J Hypertens. 2015;28(3):414-423.

24. Catena C, Colussi G, Nait F, Capobianco F, Sechi LA. Elevated homocysteine levels are associated with the metabolic syndrome and cardiovascular events in hypertensive patients. Am J Hypertens. 2015;28(7):943-950. 
25. Larijani VN, Ahmadi N, Zeb I, Khan F, Flores F, Budoff M. Beneficial effects of aged garlic extract and coenzyme Q10 on vascular elasticity and endothelial function: the FAITH randomized clinical trial. Nutrition. 2013;29(1):71-75.

26. Keiss HP, Dirsch VM, Hartung T, et al. Garlic (Allium sativum L) modulates cytokine expression in lipopolysaccharide-activated

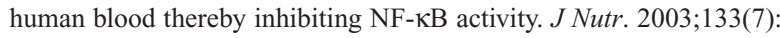
2171-2175.
27. Ide $\mathrm{N}$, Lau BH. Garlic compounds minimize intracellular oxidative stress and inhibit nuclear factor- $\mathrm{B}$ activation. J Nutr. 2001;131(3): 1020S-1026S

28. Ried K, Toben C, Fakler P. Effect of garlic on serum lipids: an updated meta-analysis. Nutr Rev. 2013;71(5):282-299.

29. Harauma A, Moriguchi T. Aged garlic extract improves blood pressure in spontaneously hypertensive rats more safely than raw garlic. J Nutr. 2006;136(3 Suppl):769S-773S

\section{Publish your work in this journal}

Integrated Blood Pressure Control is an international, peer-reviewed open-access journal focusing on the integrated approach to managing hypertension and risk reduction. Treating the patient and comorbidities together with diet and lifestyle modification and optimizing healthcare resources through a multidisciplinary team approach constitute key features of the journal. This journal is indexed on American Chemical Society's Chemical Abstracts Service (CAS). The manuscript management system is completely online and includes a very quick and fair peerreview system, which is all easy to use. Visit http://www.dovepress.com/ testimonials.php to read real quotes from published authors.

Submit your manuscript here: http://www.dovepress.com/integrated-blood-pressure-control-journal 